\title{
Permanência e Inserção de Atores na Produção Científica de 1994 a 2009 na Área de Contabilidade
}

\begin{abstract}
Resumo
Esta pesquisa buscou verificar, sob uma perspectiva longitudinal (1994-2009), o papel desempenhado pelos autores no desenvolvimento da produção científica brasileira em contabilidade. Realizou-se uma pesquisa bibliométrica e sociométrica, consultando-se 4.052 artigos. Analisaram-se categorias de produção e de continuidade, classificando-se os autores em continuantes, transientes, entrantes, one-timers e retirantes. Os resultados demonstraram a importância dos autores continuantes para o desenvolvimento, a consolidação e $\mathrm{o}$ amadurecimento da pesquisa em contabilidade. Isso porque esses autores apresentaram a maior produtividade quantitativa; atuam intermediando o relacionamento com outras categorias, ou seja, agenciam as informações de diferentes pesquisadores; os oitos autores continuantes com maior número de publicações são centrais em suas redes, articulando as pesquisas de diferentes pesquisadores; e são os principais responsáveis por parcerias internacionais. Apesar da importância dos continuantes, notouse que estes representam o menor percentual entre as categorias. Em contraposição, o maior número de autores identificados classificou-se como one-timer, ou seja, realizou uma única publicação em todo o período. Esta última categoria, aliada aos entrantes, indica a atratividade exercida sobre pesquisadores pela área do conhecimento, podendo consistir nas principais fontes de inovações e de novas abordagens. Quanto aos retirantes, observou-se certa rotatividade, o que é natural em todos os campos da pesquisa.
\end{abstract}

Palavras-chave: Produção científica em contabilidade. Permanência de autores. Internacionalização de parcerias. Bibliométrica. Sociométrica.

\author{
Silvana Anita Walter \\ Doutora em Administração (PUC/PR), \\ Professora da Universidade Regional de \\ Blumenau (FURB). Contato: Rua Antônio \\ da Veiga, Victor Konder, Blumenau, SC, \\ CEP: 89.012-900. \\ E-mail: silvanaanita.walter@gmail.com \\ Tatiana Marceda Bach \\ Bacharel em Administração (UNOESTE), \\ Mestranda em Administração pela \\ Universidade Regional de Blumenau (FURB). \\ Contato: Rua Antônio da Veiga, Victor \\ Konder, Blumenau, SC, CEP: 89.012-900. \\ E-mail: tatibach@gmail.com
}

\section{Maria José Carvalho de Souza Domingues \\ Doutora em Engenharia de produção (UFSC), Professora da Universidade Regional de Blumenau (FURB). Contato: Rua Antônio da Veiga, Victor Konder, Blumenau, SC, CEP: 89.012-900. E-mail: mjcsd2008@gmail.com}

\section{José Roberto Frega}

Doutor em Administração (PUC/PR), Professor da Universidade Federal do Paraná (UFPR). Contato: Av. Pref. Lothário Meissner, 632, Jardim Botânico, Curitiba, PR, CEP: 80210-170.

E-mail: jose.frega@gmail.com 


\section{Introdução}

As pesquisas contribuem para a produção e a disseminação do conhecimento científico (Silva \& Ensslin, 2008), e o meio mais utilizado para sua comunicação é a divulgação em congressos e periódicos (Araújo, Oliveira \& Silva, 2009). Na contabilidade, as produções científicas contribuem para um maior entendimento e difusão dos procedimentos e das técnicas relativas ao patrimônio social e econômico das entidades. Isso tornou a contabilidade, incluindo a gestão de custos, mais ativa no ambiente empresarial, contribuindo para interpretar a complexidade de informações a serem consideradas para fins decisórios (Silva \& Pires, 2009).

Nesse contexto, defende-se a relevância de analisar a produção científica em contabilidade brasileira. Essa produção é, normalmente, orientada pela bibliometria. É o que ocorre, por exemplo, com os trabalhos de Riccio, Sakata e Carastan (1999), de Oliveira (2002), de Mendonça Neto et al. (2004), de Cardoso et al. (2005), de Leite Filho (2006) e de Araújo, Oliveira e Silva (2009). Já Souza et al. (2008), Espejo et al. (2009) e Walter, Cruz, Espejo \& Gassner (2009) aliam a sociometria e a bibliometria, não investigando, contudo, a permanência dos autores e os classificando conforme critérios de produção e de continuidade. É o caso, por exemplo, de Guarido Filho, Machado-da-Silva e Gonçalves (2009), em seus estudos sobre a teoria institucional, e de Walter, Lanza, Sato, Silva \& Bach (2010), na área de estratégia. Dessa forma, acredita-se que, ao se aliar a sociometria e a classificação em categorias de produção e de continuidade, possa fornecer implicações capazes de estimular o crescimento e o amadurecimento da área.

Visando preencher a lacuna, desenvolveu-se este estudo, que buscou responder à seguinte pergunta de pesquisa: Como a produção científica brasileira, na área de contabilidade, tem se configurado quanto à permanência e à inserção de pesquisadores, bem como à cooperação entre autores? A resposta a essa pergunta envolve a verificação de como pesquisadores e instituições têm atuado no desenvolvimento da produção científica na área tanto em relação ao intercâmbio de informações (redes de coautoria) quanto à sua produção e permanência no campo.

Nesse contexto, como objetivo, definiu-se: verificar, sob uma perspectiva longitudinal (1994-2009) e de acordo com categorias de produção e de continuidade, o papel desempenhado pelos autores no desenvolvimento da produção científica brasileira em contabilidade. Para tal, realizou-se uma pesquisa bibliométrica e sociométrica, consultando-se artigos de quatro eventos brasileiros Qualis A na área contábil.

Este artigo encontra-se estruturado em cinco seções. Além desta Introdução, na segunda seção, apresenta-se a revisão de literatura com conceitos de sociometria e as categorias de continuidade. Na terceira, destacam-se os procedimentos metodológicos bibliométricos e sociométricos. A quarta destina-se à análise dos dados. Por fim, na quinta, exibem-se as considerações finais, limitações da pesquisa e sugestões para futuros estudos.

\section{Revisão de literatura}

A construção do conhecimento científico é compreendida como um processo social e dinâmico, realizada por meio das interações entre os atores do seu campo científico (Guarido Filho, Machado-da-Silva \& Gonçalves, 2009). Uma das ferramentas empregadas para estudar essa interação entre os atores de uma área é a sociometria, também conhecida como análise de redes sociais.

Para Galaskiewicz e Wasserman (1994), a análise de redes sociais concentra sua atenção em atores ou entidades sociais que interagem uns com os outros e no fato de que essas interações podem ser estudadas e analisadas como uma única estrutura ou esquema. Assim, as redes sociais, segundo Wasserman e Faust (1994), podem ser definidas como um conjunto de "nós" que correspondem a atores (pessoas ou organizações), ligados por relações sociais ou laços de tipos específicos. Em outras palavras, os processos sociais podem ser visualizados por meio de redes de relacionamentos de autoria que unem os autores ou as instituições (Walter \& Silva, 2008). Nesta pesquisa, portanto, será analisada a estrutura de relacionamento entre autores e entre instituições que se relacionam por meio da publicação conjunta de artigos na área de contabilidade. Essa possibilidade de análise é chamada de análise de redes de coautoria (Liu, Bollen, Nelson \& Van de Sompel, 2005). 
Para Powel, Koput e Smith-Doerr (1996) e Stuart e Podolny (1999), a conexão em uma rede, além de aumentar o acesso à informação, é uma oportunidade para o acesso à inovação por meio de conhecimentos gerados pelos relacionamentos individuais. Sob um ponto de vista institucional, conforme destacam Smitt-Doerr e Powell (2003), as redes governam a distribuição e o acesso aos recursos e às informações, de forma que as conexões podem conduzir ao fortalecimento de atividades, às oportunidades e à aprendizagem. Contudo, quando esse acesso é restrito, pode proporcionar um fechamento social. No caso das redes de coautoria, essas conexões tornam-se oportunidades para o intercâmbio de informações e ideias que se efetuam no desenvolvimento de novas pesquisas, as quais podem contribuir para o desenvolvimento da área de conhecimento.

Alguns conceitos da sociometria são importantes para análise da cooperação entre autores e instituições em uma área de pesquisa. Os "nós", por exemplo, correspondem a cada ator que colabora com, pelo menos, um dos itens de uma rede e se caracterizam por círculos de diferentes cores em uma rede (Walter et al., 2010). Os nós nesta pesquisa referem-se aos pesquisadores ou às instituições aos quais os autores dos artigos vinculavam-se no momento da publicação.

Laço forte é a conexão direta dos atores em uma rede (Granovetter, 1973), na qual as informações a serem compartilhadas tendem a ser as mesmas, com baixa tendência para mudança (Burt, 1992). No caso deste estudo, o laço forte refere-se a dois autores (pesquisadores ou instituições) que publicaram um artigo em parceria. Por sua vez, laço fraco é a representação de contatos indiretos formados por meio de pontes que fornecem diferentes fontes de informação e tornam a rede propensa à inovação (Granovetter, 1973). Nesse sentido, no caso das redes de cooperação entre autores, os laços fracos representam laços indiretos, operacionalizados por meio da interação entre um autor que publica com outros pesquisadores. Lacuna estrutural representa contatos não conectados em uma rede, o que fornece uma vantagem competitiva para o indivíduo que realiza a conexão entre as diferentes redes (Burt, 1992). Assim, um autor que estabelece a conexão entre redes detém o poder de agenciamento do contato entre os autores dos diferentes grupos aos quais se encontra vinculado.

A densidade de uma rede, para Marsden (1993), reflete quantos atores desta rede estão conectados uns aos outros, de forma que quanto maior o número de laços fortes entre os atores da rede, maior sua densidade. O conceito de equivalência estrutural, desenvolvido por White, Boorman e Breiger (1974) e Burt (1992), ocorre quando dois atores ocupam posições semelhantes em um sistema social, possuindo os mesmos tipos de relações. A propriedade de centralidade dos atores em uma rede, por seu turno, reflete sua importância nessa rede, sendo que, quanto mais centrais, mais importantes os autores serão (Wasserman \& Faust, 1994). De acordo com Knoke (1990), a posição do ator na rede possui o poder de influenciar as atitudes e os comportamentos de outros atores a partir de sua proeminência na rede, na qual a informação e os recursos escassos são transferidos de um ator para outro.

Para o estabelecimento de uma rede bem estruturada de relacionamentos entre autores de um campo do conhecimento, necessita-se de certo grau de permanência no âmbito da produção científica. Essa permanência, de acordo com Guarido Filho, Machado-da-Silva e Gonçalves (2009), é sustentada por um grupo de pesquisadores que se articulam e cooperam no sentido de conformar e adotar um quadro de referência para a área de conhecimento. Na concepção de Shah (2000), os atores que permanecem por um amplo tempo em uma rede, tornam-se centrais nesta. Walter et al. (2010) acrescentam que a permanência de pesquisadores em um campo favorece o desenvolvimento e o amadurecimento da área por meio do emprego do conhecimento que esses detêm, assim como a entrada de novos pesquisadores pode ser importante para introduzir novos conhecimentos, abordagens e visões.

Um meio utilizado para analisar a permanência dos pesquisadores em uma área do conhecimento é a classificação em categorias de produção e de continuidade (Braun, Glãnzel \& Schubert, 2001; Gordon, 2007; Guarido Filho et al., 2009; Walter et al., 2010), sendo que os autores são classificados de acordo com a regularidade e a distribuição de suas publicações ao longo do período. Os pesquisadores considerados como continuantes são os que apresentam diferentes publicações em distintos períodos do tempo, inclusive recentemente, ou seja, são os integrantes habituais de um campo no qual atuam há um tempo considerável 
(Walter et al., 2010). Esses autores costumam se apresentar em um número reduzido se comparado a outras categorias, todavia tendem a ser os mais produtivos, o que aponta para o fato de que um número menor de pesquisadores é responsável pelo maior número de publicações em uma área ou tema estudo (Guarido Filho et al., 2009). Esses pesquisadores também podem atuar na intermediação entre outras categorias e entre pesquisadores em uma rede, ou seja, costumam se apresentar como autores centrais em suas redes.

Os transientes diferem dos continuantes por apresentarem publicações em um número mais restrito, sendo, portanto, um pouco menos persistentes e estáveis que aqueles (Walter et al., 2010). Apesar de menos persistentes, os transientes costumam apresentar um papel similar aos dos continuantes em uma área do conhecimento, de forma que estas duas categorias, continuantes e transientes, são apontados como essenciais para a sustentação e a continuidade da pesquisa em um campo em estudo (Guarido Filho et al., 2009).

Os one-timers, em contraposição, são os autores esporádicos da área, apresentando apenas uma publicação no período analisado (Walter et al., 2010). Guarido Filho, Machado-da-Silva e Gonçalves (2009) destacam que é provável que essas publicações sejam fruto de dissertações de mestrado com orientação de pesquisadores mais estabelecidos no campo ou oriundas de pesquisadores concentrados em outras áreas, mas que em algum momento visualizaram a possibilidade de publicar também no campo em estudo. Tem-se ainda que parte desses pesquisadores, no futuro, poderia ser reclassificada como entrantes ou transientes, caso venham a publicar novamente na área (Guarido Filho et al., 2009).

Entrantes são os que começaram a integrar o campo há pouco tempo, exibindo publicações apenas nos períodos mais atuais. Esses pesquisadores representam o quanto a área pesquisada é atrativa para novos pesquisadores, bem como a possibilidade de inovações e transformações na área oriundas de pesquisadores com novas ideias e perspectivas (Walter et al., 2010).

Por fim, os retirantes consistem naqueles que deixaram a área, ou seja, não apresentaram publicações nos últimos anos (Walter et al., 2010). Eles podem ser tanto pesquisadores que estão em processo de desligamento de suas vidas acadêmicas, diminuindo o ritmo de publicações, quanto pesquisadores que estão migrando para outros campos do conhecimento.

\section{Delineamento metodológico}

Para atender ao objetivo apresentado, realizou-se uma pesquisa bibliométrica que, segundo Macias-Chapula (1998), consiste no estudo dos aspectos quantitativos da produção, da disseminação e do uso da informação registrada; e sociométrico, que explora a matriz de relacionamentos estabelecida entre atores sociais (Galaskiewicz \& Wasserman, 1994), aqui compreendidos como autores e instituições. Quanto à perspectiva temporal, esta pesquisa caracteriza-se como longitudinal, considerando o período de 16 anos: de 1994 a 2009.

Por meio de pesquisa documental, coletou-se um total de 4.052 artigos científicos, abrangendo as publicações em eventos Qualis A (até 2009) do país, na área contábil: a) International Accouting Congress, da Associação Nacional dos Programas de Pós-Graduação em Ciências Contábeis (IAAER-Anpcont), no período de 2007 a 2009, totalizando 346 artigos; b) Congresso Brasileiro de Custos (CBC), do período de 1994 a 2009, em todas as suas 16 edições, totalizando 2.530 artigos; c) Congresso USP de Controladoria e Contabilidade, no período de 2001 a 2009, totalizando 473 artigos; e d) Encontro da Associação Nacional de Pós-Graduação e Pesquisa em Administração (EnANPAD), no período de 1998 a 2009, em suas áreas temáticas de Contabilidade (CON) e Ensino e Pesquisa em Contabilidade (EPQ), totalizando 703 artigos. Selecionaram-se esses quatro eventos por serem classificados como nível "A" pela Coordenação de Aperfeiçoamento de Pessoal de Nível Superior (CAPES) e por sua importância e representatividade no cenário nacional.

Para a análise dos dados, observaram-se o ano de publicação, o evento no qual foram publicados, os autores dos artigos, a primeira instituição informada na qual os autores se encontravam vinculados na ocasião da publicação e o país de origem da instituição. Para garantir a diferenciação nos nomes dos auto- 
res que apresentavam a mesma forma de citação nominal, procedeu-se à conferência individual por meio de uma consulta ao currículo constante na Plataforma Lattes, do Conselho Nacional de Desenvolvimento Científico e Tecnológico (CNPq), e, após a averiguação de possíveis homônimos, optou-se, nesses casos, por manter o sobrenome e destacar o primeiro nome por extenso.

Realizou-se a análise dos dados no tocante: à produção científica por evento e por ano; à classificação dos pesquisadores conforme as categorias de produção e de continuidade; à produção científica por categoria e por ano; à produção científica por evento em cada categoria; às coautorias entre categorias; aos autores mais prolíficos e com maior número de laços; às redes sociais de cooperação entre atores; aos laços por categoria dos autores mais prolíficos; às redes sociais de cooperação entre instituições brasileiras e estrangeiras; às instituições brasileiras que se associaram a estrangeiras; e às categorias de produção e de continuidade dos autores que realizaram parcerias internacionais.

Para a análise das categorias de produção e de continuidade, avaliou-se quantitativamente o volume (absoluto e relativo) de artigos, de pesquisadores e de autorias presentes em cada ano analisado. Assim, classificaram-se, a partir dos critérios apresentados por Walter et al. (2010) - adaptados de Braun, Glänzel e Schubert (2001), Gordon (2007) e Guarido Filho, Machado-da-Silva e Gonçalves (2009) -, os pesquisadores como: (a) continuantes: com duas ou mais publicações em cinco ou mais anos diferentes e, ao menos, uma nos últimos três anos; (b) transientes: com duas ou mais publicações em até quatro anos diferentes (não mais), sendo, pelo menos, uma nos três últimos anos e, ao menos, uma em anos anteriores; (c) one-timers: com apenas uma publicação no período analisado; (d) entrantes: com duas ou mais publicações em um ou mais anos diferentes nos últimos três anos exclusivamente; e (e) retirantes: com duas ou mais publicações em um ou mais anos diferentes, mas sem publicação nos últimos três anos. Ressalta-se que essas categorias são mutuamente excludentes como pode ser percebido a partir da definição e dos critérios de cada categoria expostos na Figura 1.

\begin{tabular}{|c|c|c|}
\hline Categoria & Definição & Critérios para classificação \\
\hline \multirow{2}{*}{ Entrantes } & \multirow{2}{*}{$\begin{array}{l}\text { Novos pesquisadores da área (publicaram, pelo } \\
\text { menos, } 2 \text { artigos apenas nos últimos } 3 \text { anos) }\end{array}$} & $\geq 2$ artigos de 2007 a 2009 \\
\hline & & Sem publicações de 1994 a 2006 \\
\hline \multirow{3}{*}{ Transientes } & \multirow{3}{*}{$\begin{array}{l}\text { Pesquisadores relativamente permanentes na área } \\
\text { (publicaram, pelos menos, dois artigos em até } 4 \text { anos } \\
\text { diferentes, tanto nos } 3 \text { últimos quanto antes disso) }\end{array}$} & $\geq 2$ artigos em até 4 anos diferentes \\
\hline & & $\geq 1$ artigos de 2007 a 2009 \\
\hline & & $\geq 1$ artigos de 1994 a 2006 \\
\hline \multirow[b]{2}{*}{ Continuantes } & \multirow{2}{*}{$\begin{array}{l}\text { Pesquisadores consolidados na área (publicaram, } \\
\text { pelos menos, dois artigos em cinco ou mais anos } \\
\text { diferentes, incluindo os últimos } 3 \text { anos) }\end{array}$} & $\geq 2$ artigos em $\leq 5$ anos \\
\hline & & $\geq 1$ artigos de 2007 a 2009 \\
\hline One-timers & $\begin{array}{l}\text { Pesquisadores esporádicos (publicaram apenas um } \\
\text { artigo em todo o período) }\end{array}$ & 1 artigo de 1994 a 2009 \\
\hline \multirow{2}{*}{ Retirantes } & \multirow{2}{*}{$\begin{array}{l}\text { Pesquisadores que estão deixando a área (publicaram, } \\
\text { pelos menos, } 2 \text { artigos, mas nenhum nos últimos } 3 \text { anos) }\end{array}$} & $\geq 2$ artigos de 1994 a 2006 \\
\hline & & Sem publicações de 2007 a 2009 \\
\hline
\end{tabular}

Figura 1. Definição e critérios para classificação dos autores nas categorias de produção e continuidade.

Fonte: Adaptado de Guarido Filho, Machado-da-Silva e Gonçalves (2009).

Quanto à análise das redes sociais, optou-se pela exploração das redes de coautoria entre autores e entre instituições, o que representa uma vertente de análise de redes sociais (Liu et al., 2005), por meio do software $\mathrm{UCINET}^{\circ}$ 6, com base no ano de publicação dos artigos analisados. Para a contagem de laços por autores e instituições, considerou-se cada associação como um laço. 


\section{Apresentação e análise dos dados}

Nesta seção, expõe-se a análise da produção científica na área de contabilidade nos últimos 16 anos. Primeiramente, na Tabela 1, apresenta-se a quantidade de artigos publicados, por ano e por evento, considerada nesta pesquisa.

Tabela 1

Quantidade de artigos publicados por ano, por evento e total

\begin{tabular}{|c|c|c|c|c|c|c|c|c|c|c|c|c|c|c|c|c|c|}
\hline Eventos & ুั & ผั & ஜ & ริ & ஃ̊ & รั & ¿্่ & চ্் & ণั & ஜ̊ํ & ষ্ণ & 옹 & ஜํ & 오 & $\stackrel{\infty}{\circ}$ & 유 & Total \\
\hline \multirow{2}{*}{ ANPCONT } & \multirow{2}{*}{\multicolumn{4}{|c|}{-}} & \multirow{2}{*}{\multicolumn{3}{|c|}{-}} & \multirow{2}{*}{\multicolumn{3}{|c|}{-}} & \multirow{2}{*}{\multicolumn{3}{|c|}{-}} & 47 & 95 & 204 & \multirow{2}{*}{346} \\
\hline & & & & & & & & & & & & & & \multicolumn{3}{|c|}{$23,25 \%(346)$} & \\
\hline \multirow{2}{*}{$\mathrm{CBC}$} & 27 & 81 & 42 & 49 & 76 & 106 & 139 & 133 & 179 & 139 & 238 & 351 & 212 & 238 & 267 & 253 & \multirow{2}{*}{2.530} \\
\hline & \multicolumn{4}{|c|}{$100 \%$ (199) } & \multicolumn{3}{|c|}{$85,6 \%(321)$} & \multicolumn{3}{|c|}{$53,56 \%(451)$} & \multicolumn{3}{|c|}{$69,77 \%(801)$} & \multicolumn{3}{|c|}{$50,94 \%(758)$} & \\
\hline \multirow{2}{*}{$\begin{array}{l}\text { Congresso } \\
\text { USP }\end{array}$} & \multirow{2}{*}{\multicolumn{4}{|c|}{ - }} & \multirow{2}{*}{\multicolumn{3}{|c|}{-}} & 74 & 85 & 101 & 33 & 30 & 35 & 30 & 44 & 41 & \multirow{2}{*}{473} \\
\hline & & & & & & & & \multicolumn{3}{|c|}{$30,88 \%(260)$} & \multicolumn{3}{|c|}{$8,54 \%(98)$} & \multicolumn{3}{|c|}{$7,73 \%(115)$} & \\
\hline \multirow{2}{*}{ EnANPAD } & \multirow{2}{*}{\multicolumn{4}{|c|}{-}} & 12 & 10 & 32 & 24 & 47 & 60 & 78 & 82 & 89 & 112 & 90 & 67 & \multirow{2}{*}{703} \\
\hline & & & & & \multicolumn{3}{|c|}{$14,4 \%(54)$} & \multicolumn{3}{|c|}{$15,56 \%(131)$} & \multicolumn{3}{|c|}{$21,69 \%$ (249) } & \multicolumn{3}{|c|}{$18,08 \%$ (269) } & \\
\hline \multirow{2}{*}{ Total } & 27 & 81 & 42 & 49 & 88 & 116 & 171 & 231 & 311 & 300 & 349 & 463 & 336 & 427 & 496 & 565 & 050 \\
\hline & \multicolumn{4}{|c|}{199} & \multicolumn{3}{|c|}{375} & \multicolumn{3}{|c|}{842} & \multicolumn{3}{|c|}{1.148} & \multicolumn{3}{|c|}{1.488} & +.052 \\
\hline
\end{tabular}

A partir da Tabela 1, verifica-se que, em geral, há uma expansão quantitativa em número de artigos publicados ao longo dos anos. Nota-se, também, a criação de novos eventos na área de contabilidade, como o Congresso USP, em 2001, e o Anpcont, em 2007. O CBC é o primeiro evento a compor o universo do estudo, desde 1994, também se destacando pelo maior número de artigos publicados por edição e no total.

A Tabela 2 apresenta a distribuição de pesquisadores segundo as categorias.

Tabela 2

\section{Distribuição de pesquisadores segundo categorias de produção e de continuidade}

\begin{tabular}{lcccc}
\hline $\begin{array}{l}\text { Categorias de } \\
\text { produção e de } \\
\text { continuidade }\end{array}$ & $\begin{array}{c}\text { Autores } \\
\text { independentemente } \\
\text { do número de } \\
\text { publicações }\end{array}$ & $\begin{array}{c}\text { Autores } \\
\text { considerando } \\
\text { o número de } \\
\text { publicações }^{2}\end{array}$ & Artigos $^{3}$ & $\begin{array}{c}\text { Períodos com } \\
\text { produção }^{4}\end{array}$ \\
\hline Continuantes & $8,2 \%(400)$ & 3.067 & $75,7 \%(3.067)$ & $100,0 \%(16)$ \\
\hline Transientes & $13,9 \%(679)$ & 1.526 & $37,7 \%(1.526)$ & $100,0 \%(16)$ \\
\hline One-Timers & $50,8 \%(2.478)$ & 2.478 & $61,2 \%(2.478)$ & $100,0 \%(16)$ \\
\hline Entrantes & $10,0 \%(488)$ & 984 & $24,3 \%(984)$ & $18,7 \%(03)$ \\
\hline Retirantes & $17,1 \%(835)$ & 1.731 & $42,7 \%(1.731)$ & $81,3 \%(13)$ \\
\hline Total & 4.880 & 9.786 & 4.052 & 16 \\
\hline
\end{tabular}

Como muitos artigos possuem mais de um ator, existindo, portanto, a possibilidade de os autores de um mesmo artigo se enquadrarem em diferentes categorias, adotaram-se alguns critérios de quantificação:

${ }^{1}$ Considera o número de autores que podem ser classificados em cada categoria sem repetição do autor, ou seja, cada autor é contabilizado apenas uma vez, independente do número de publicações. O percentual é obtido em relação ao número total de autores diferentes no período (4.880).

${ }^{2}$ Considera o número de vezes em que os autores classificados em cada categoria publicaram, ou seja, admite repetição do mesmo autor conforme o número de publicações suas no período.

${ }^{3}$ Considera o número de artigos em que os autores classificados em cada categoria contribuíram como autores ou coautores. A obtenção do percentual ocorre em relação ao número de artigos publicados (4.052).

${ }^{4}$ Considera o número de anos, de 1994 a 2009, em que houve artigo(s) publicado(s) pelos autores classificados em cada categoria. Obtém-se o percentual em relação ao número total de anos no período (16). 
Observa-se, por meio da Tabela 2, que os autores continuantes representam, ao longo de 16 anos, $8,2 \%$ do total de autores. Esses pesquisadores aparecem 3.067 vezes como autores ou coautores de estudos e representam $75,4 \%$ do volume de produção na área. Esse percentual de continuantes é mais amplo que o encontrado em áreas mais específicas de conhecimento, como de 5,5\% na perspectiva institucional em estudos organizacionais (Guarido Filho et al., 2009) e de 2,9\% em estratégia organizacional (Walter et al., 2010). Dessa forma, percebe-se que a área de contabilidade como um todo apresenta maior percentual de pesquisadores tradicionais, que mantêm constância e representatividade em suas publicações. Se esse resultado também se aplicar a áreas mais específicas da contabilidade, poderá ser objeto de estudo futuro.

Os autores continuantes são responsáveis por um índice de produtividade quantitativa (divisão do número de artigos pelo número de autores independente das publicações) de 7,67, superior aos transientes, que obtiveram 2,25; aos retirantes, com 2,07; e aos entrantes, com 2,02. Os continuantes se destacam por contribuírem para a consolidação do conhecimento da área, haja vista sua experiência e conhecimento a respeito dos temas relacionados à área contábil. Além disso, também contribuem, por meio de sua permanência e ampla produtividade, para difusão e legitimação do conhecimento praticado no campo.

Os transientes, segunda categoria que apresenta maior estabilidade em publicações, somaram 13,9\% do total de autores, envolvendo 679 pesquisadores que se evidenciaram 1.526 vezes em autoria ou coautorias de artigos. Os percentuais de continuantes e de transientes atingem, somados, $22,1 \%$ dos autores, porém com um índice de produtividade médio de 4,96. Essas duas categorias de autores em conjunto, de acordo com Guarido Filho, Machado-da-Silva e Gonçalves (2009), representam as bases de sustentação e de continuidade da pesquisa em um campo de estudo.

Um dado a se destacar é o número de one-timers, que totalizou 50,8\% dos diferentes autores. Esse número é inferior ao encontrado por Guarido Filho, Machado-da-Silva e Gonçalves (2009), de 59,8\%, e por Walter et al. (2010), de 72,9\%, mas ainda merece atenção, visto que esses pesquisadores analisaram áreas mais específicas do conhecimento. Os one-timers, para Guarido Filho, Machado-da-Silva e Gonçalves (2009), podem ser pesquisadores com interesse predominante em outra área, tendo contribuído pontualmente para o campo em análise. Aliados aos entrantes (10\% dos autores), os one-timers também representam o poder de atração desempenhado pela área, dado que pesquisadores sem histórico de publicações no campo de contabilidade estão submetendo seus artigos em eventos desse campo.

Comparando-se os entrantes ( $10 \%$ dos autores que participaram de $24,2 \%$ das publicações) e retirantes (17,1\% dos autores que participaram de $42,7 \%$ das publicações), observa-se que o número de autores que deixa a área e sua produtividade é mais amplo do que os que nela se inserem. Isso pode representar um retrocesso no desenvolvimento quantitativo da área, caso não se leve em consideração que parte dos autores classificados como one-timers pode se configurar como entrantes nos próximos anos.

A Tabela 3 apresenta a quantidade de artigos publicados por categoria e por ano. Esta tabela foi construída de modo que os autores são primeiramente classificados em uma das categorias de produção e continuidade. Na sequência, é somado o número de publicação de todos os autores de cada categoria em cada ano. 
Tabela 3

Artigos publicados por categoria e por ano

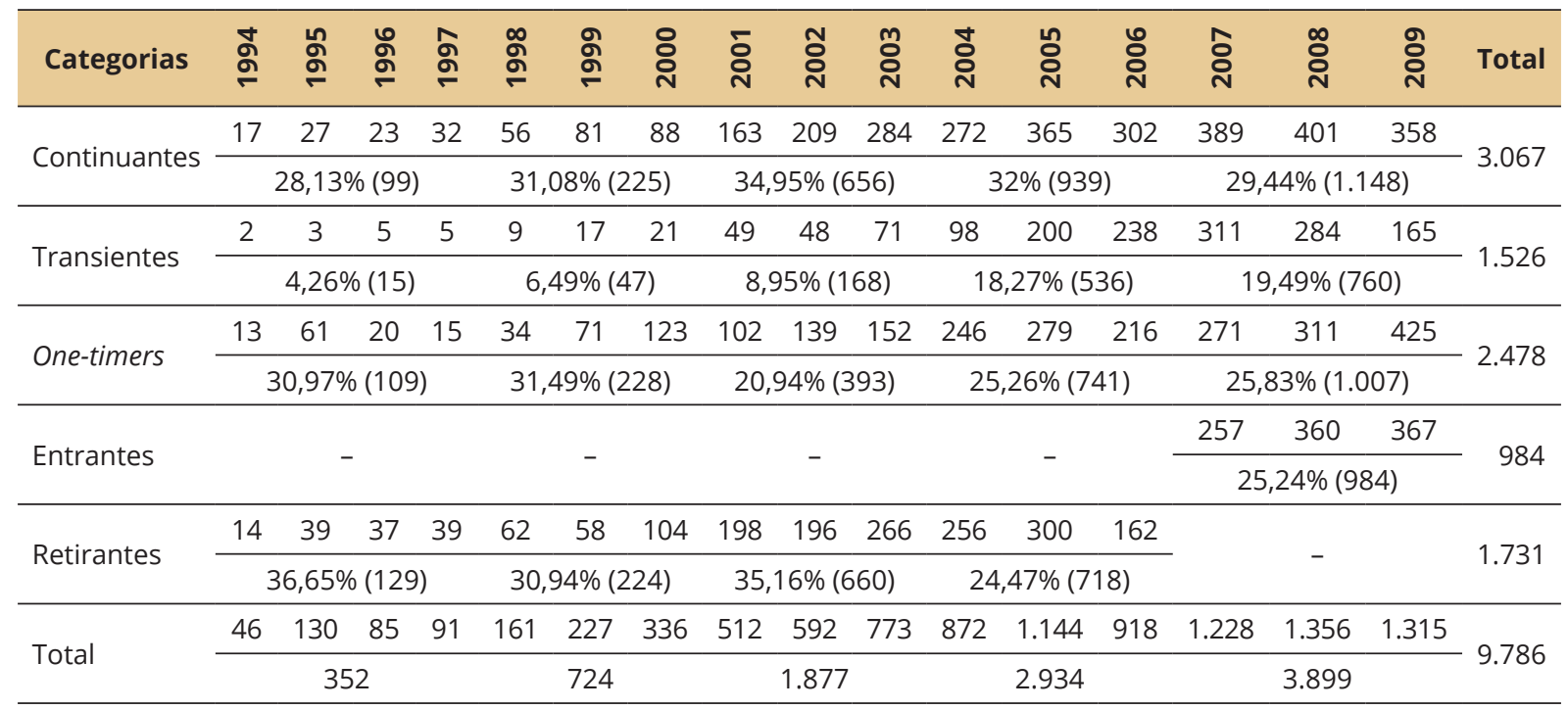

No que concerne à Tabela 3, o período de 1994-1997 se destaca pelos menores índices de continuantes e transientes, bem como o segundo maior de one-timers. Assim, tem-se um primeiro período com destaque para publicações de autores esporádicos e que estão deixando a área. O período de 1998-2000 possui o segundo menor percentual de autores transientes e um percentual similar de continuantes, de one-timers e de retirantes, apresentando-se como um período equilibrado. Em 2001-2003, exibe-se o segundo maior percentual de retirantes, acima do período anterior. Esse mesmo período também apresenta o maior percentual de continuantes e o menor de one-timers, configurando-se como um período de destaque para autores tradicionais e que estão deixando a área. Em 2004-2006, observa-se o início de uma elevação no percentual de transientes e uma queda no de retirantes, aspecto que tem continuidade no período de 2007-2009. Assim, no último período, tem-se o maior percentual de transientes e o aparecimento dos entrantes. Nesse sentido, destaca-se que os entrantes de 2007-2009 poderão se tornar transientes, continuantes ou retirantes no futuro, assim como os one-timers dos últimos períodos poderão se tornar entrantes no futuro (Guarido Filho et al., 2009).

Na Tabela 4, exibe-se a quantidade de artigos publicados por evento por categoria.

Tabela 4

\section{Artigos publicados por evento em cada categoria de produção e de continuidade}

\begin{tabular}{lcccc}
\multicolumn{1}{c}{ Categorias } & ANPCONT & Congresso USP & \multicolumn{1}{c}{ CBC } & EnANPAD \\
\hline Continuantes & $34,10 \%(179)$ & $34,38 \%(384)$ & $28,60 \%(1.830)$ & $38,62 \%(674)$ \\
\hline Transientes & $16,00 \%(84)$ & $15,76 \%(176)$ & $14,96 \%(957)$ & $17,71 \%(309)$ \\
\hline One-timers & $24,57 \%(129)$ & $22,20 \%(248)$ & $27,33 \%(1.749)$ & $20,17 \%(352)$ \\
\hline Entrantes & $25,33 \%(133)$ & $6,80 \%(76)$ & $9,38 \%(600)$ & $10,03 \%(175)$ \\
\hline Retirantes & - & $20,86 \%(233)$ & $19,74 \%(1.263)$ & $13,47 \%(235)$ \\
\hline
\end{tabular}


A Tabela 4 permite observar que, em todos os eventos, o percentual de continuantes é maior do que das demais categorias, sendo que, no EnANPAD, esse é mais amplo que nos outros eventos. Ainda no tocante ao EnANPAD, observa-se que, somando-se os percentuais de continuantes e de transientes, categorias mais estáveis em publicações na área, tem-se mais de 50\% dos artigos. Esse evento também apresenta o menor percentual de one-timers. Assim, se configura como o evento com publicação de pesquisadores mais tradicionais.

Em contraposição, o CBC apresenta o maior percentual de one-timers, apresentando-se como o evento com maior percentual de pesquisadores esporádicos. Esse resultado pode estar relacionado à amplitude de artigos aceitos, o que pode ser um atrativo para novos autores que ainda não publicaram na área. Outra possibilidade é a de que, por enfocar a área de gestão estratégica de custos, o evento atraia, também, pesquisadores de áreas como administração e economia, os quais publiquem, possivelmente, em outros eventos distintos aos pesquisados neste estudo. Essa segunda possibilidade atribui ao evento um caráter mais interdisciplinar, possibilitando a compilação de contribuições de diferentes áreas para o tema.

O Anpcont se destaca pelo percentual de entrantes, resultado possivelmente influenciado por ser o evento mais recente do universo pesquisado. Os percentuais somados de entrantes e one-timers (que poderão se tornar entrantes, já que o evento possui poucas edições até o momento) do Anpcont atinge quase $50 \%$. Esse percentual se configura como forte indício de inovação, por ser um importante meio de inserção de novos pesquisadores e, possivelmente, de perspectivas para a contabilidade. Já o Congresso USP, em contrapartida, apresentou, entre os eventos enfocados, o menor percentual de entrantes apesar de ser o segundo mais recente.

Em uma análise a respeito das coautorias relacionadas a cada categoria de produção e de continuidade, verificou-se que os continuantes publicam principalmente com on-timers (939), mas também com transientes (765), retirantes (671) e entrantes (509). A maior publicação dos continuantes com on-timers pode estar relacionada ao fato de que essas publicações de on-timers podem ser fruto de dissertações de mestrado com orientação de outros pesquisadores já estabelecidos no campo (continuantes), conforme apontam Guarido Filho, Machado-da-Silva e Gonçalves (2009). Também pode ser resultado de outras atividades dos programas de pós-graduação, como publicações advindas de disciplinas isoladas ou de participações temporárias em grupos de pesquisas, o que reflete a transição desses autores (Walter et al., 2010).

Os transientes publicaram com continuantes (890), on-timers (647), entrantes (292) e retirantes (277). Já os on-timers publicaram com continuantes (917), transientes (610), retirantes (490) e entrantes (331). Os entrantes, por sua vez, publicaram com continuantes (490), one-timers (311) e transientes (263). Por fim, os retirantes publicaram com continuantes (693), one-timers (519) e transientes (233). Assim, transientes, one-timers, entrantes e retirantes publicam principalmente com continuantes. Esses números permitem inferir que os continuantes atuam intermediando relacionamento com diferentes categorias, $o$ que corrobora a afirmação de Braun, Glanzel e Schubert (2001) de que os continuantes exercem importante papel na construção do conhecimento, especialmente na consolidação da produção e na articulação com outras categorias de autores. Isso pode indicar uma maior influência dos continuantes sobre o fluxo e conteúdo das informações na área. As publicações entre continuantes e transientes, por exemplo, apontam para uma maior possibilidade de associações duradouras no campo, além de que os continuantes estão atraindo entrantes e one-timers que poderão se tornar entrantes para a área.

Na Tabela 5, apresentam-se os 25 autores com maior número de publicações no período. 
Tabela 5

\section{Autores mais prolíficos}

\begin{tabular}{|c|c|c|c|c|c|c|c|c|c|c|c|c|c|c|c|c|c|c|c|c|}
\hline \multirow[b]{2}{*}{ Autores } & \multicolumn{16}{|c|}{ Artigos publicados } & \multirow{2}{*}{ 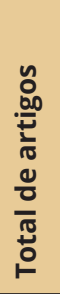 } & \multirow[b]{2}{*}{ 芯 } & \multirow[b]{2}{*}{ 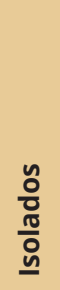 } & \multirow{2}{*}{ 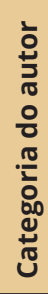 } \\
\hline & ઠั & ฝั & ুั & ڤે & $\stackrel{\infty}{\circ}$ & ু & ঃి & চ্ণ & ర్ & @్̊ & ષั & ๕̊ํํ & ষ্ঠ & ¿̊ํํ & ֻ̊ํํ & ஓ्̀ & & & & \\
\hline BEUREN, I. M. & - & - & 1 & 2 & 3 & 1 & 1 & 7 & 6 & 6 & 4 & 4 & 4 & 7 & 6 & 6 & 58 & 84 & 3 & $\mathrm{C}^{2}$ \\
\hline MIRANDA, L. C. & - & - & - & - & - & 1 & 4 & 7 & 15 & 5 & 2 & 2 & - & 2 & 3 & 4 & 45 & 108 & 1 & $\mathrm{C}$ \\
\hline CORRAR, L. J. & - & - & - & 1 & 1 & 3 & 1 & - & 3 & 5 & 4 & 1 & 2 & 5 & 3 & 9 & 38 & 64 & 2 & $\mathrm{C}$ \\
\hline SOUZA, M. A. de & - & - & - & - & - & - & - & 2 & 2 & 3 & 3 & 6 & 3 & 6 & 5 & 5 & 35 & 60 & 1 & $\mathrm{C}$ \\
\hline SOUZA, A. A. de & - & - & - & - & 1 & - & 2 & 2 & 3 & 3 & 4 & 5 & 3 & 4 & 3 & 5 & 35 & 98 & - & C \\
\hline SILVA, C. A. T. & - & 1 & 1 & 1 & 2 & 1 & 2 & 4 & 2 & 5 & 2 & 5 & 3 & 3 & 1 & 2 & 35 & 52 & 6 & $C$ \\
\hline GUERREIRO, R. & - & 1 & 1 & - & 2 & 2 & 1 & - & 4 & 5 & 3 & 3 & 3 & 3 & 4 & 3 & 35 & 51 & - & $\mathrm{C}$ \\
\hline BORNIA, A. C. & 2 & 2 & 1 & 2 & - & 5 & 2 & 6 & 1 & - & 2 & 3 & 2 & 1 & 3 & 2 & 34 & 55 & 3 & $\mathrm{C}$ \\
\hline CALLADO, A. A. C. & - & - & - & - & 1 & 2 & 2 & 1 & 8 & 1 & 1 & 4 & 2 & 3 & 4 & 1 & 30 & 55 & - & $\mathrm{C}$ \\
\hline OTT, E. & - & - & - & - & - & - & - & 3 & 3 & 3 & 2 & 4 & 3 & 3 & 3 & 6 & 30 & 47 & 2 & $C$ \\
\hline MACEDO, M. A.da S. & - & - & - & - & - & - & - & - & - & 1 & 3 & 3 & 4 & 5 & 7 & 7 & 30 & 51 & - & $\mathrm{C}$ \\
\hline CALLADO, A. L. C. & - & - & - & - & 1 & 1 & 1 & 1 & 7 & 1 & 1 & 5 & 3 & 3 & 4 & 1 & 29 & 57 & - & $\mathrm{C}$ \\
\hline WERNKE, R. & - & - & - & - & - & 3 & 1 & 4 & 3 & 1 & 3 & 3 & 3 & 2 & 2 & 3 & 28 & 29 & - & $\mathrm{C}$ \\
\hline NOSSA, V. & - & - & - & - & 1 & 1 & 2 & - & 1 & 7 & 5 & 3 & - & 3 & 1 & 4 & 28 & 59 & 1 & $C$ \\
\hline PEREIRA, C. A. ${ }^{1}$ & - & - & - & - & - & 1 & - & - & - & 3 & 7 & 3 & 4 & 4 & 4 & 1 & 27 & 51 & - & $\mathrm{C}$ \\
\hline TEIXEIRA, A. J. C. & - & - & - & - & - & - & - & - & 1 & 9 & 2 & 4 & - & 4 & 2 & 4 & 26 & 63 & - & $C$ \\
\hline SLOMSKI, V. & - & - & - & - & - & - & - & - & - & 7 & 3 & 4 & 3 & 5 & 2 & 2 & 26 & 51 & - & $\mathrm{C}$ \\
\hline DIEHL, C. A. & - & - & - & - & - & 1 & 1 & - & 3 & 1 & 2 & 2 & 1 & 6 & 4 & 5 & 26 & 34 & 7 & $\mathrm{C}$ \\
\hline BORBA, J. A. & - & - & - & 1 & - & - & 1 & - & - & 2 & 4 & 4 & 4 & 2 & 4 & 3 & 25 & 42 & 1 & $C$ \\
\hline FREZATTI, F. & - & - & - & - & 1 & 1 & 1 & - & 1 & 1 & 1 & 3 & 1 & 5 & 7 & 3 & 25 & 48 & 3 & $\mathrm{C}$ \\
\hline NASCIMENTO, A.M. & - & - & - & - & - & - & - & - & - & 2 & 3 & 4 & 6 & 7 & 2 & 1 & 25 & 47 & - & $\mathrm{C}$ \\
\hline BACIC, M. J. & 2 & 2 & 2 & 2 & 2 & 1 & 1 & 1 & 2 & 1 & 2 & 2 & 1 & 1 & 2 & - & 24 & 28 & 4 & $C$ \\
\hline KASSAI, J. R. & - & - & - & - & 2 & 3 & 3 & 3 & 1 & 3 & 3 & 2 & - & 2 & 2 & - & 24 & 41 & 5 & $\mathrm{C}$ \\
\hline FREIRE, F. de S. & - & 1 & & 1 & 2 & 2 & - & 4 & 2 & 1 & 2 & 1 & 2 & 2 & 1 & 3 & 24 & 49 & 2 & $\mathrm{C}$ \\
\hline MARTINEZ, A. L. & - & - & - & - & 1 & 2 & - & - & - & 2 & 4 & 2 & 5 & 2 & 2 & 4 & 24 & 18 & 10 & $\mathrm{C}$ \\
\hline 'PEREIRA, Carlos & & & & & & & & & & . & те & entrite & & & & & & & & \\
\hline
\end{tabular}

Nota-se, a partir da Tabela 5, que César A. T. Silva e Miguel J. Bacic tiveram artigos publicados em 15 dos 16 anos analisados, seguidos por Ilse M. Beuren e Antonio C. Bornia, com publicações em 14 anos diferentes. Ilse M. Beuren também se destaca como a autora mais prolífica, seguida por Luiz C. Miranda, o qual se apresenta como o autor com maior número de laços de cooperação. Referente à publicação de artigos como único autor, tem-se Antonio L. Martinez com o maior número. Tem-se, ainda, que todos os autores mostrados na Tabela 5 classificam-se como continuantes.

Na Figura 2, destacam-se as redes de cooperação dos oito autores com maior número de publicações no período analisado. Ressalta-se que se empregou esse recorte metodológico diante da impossibilidade de representar as redes de cooperação entre todos os autores por limitações de espaço. 


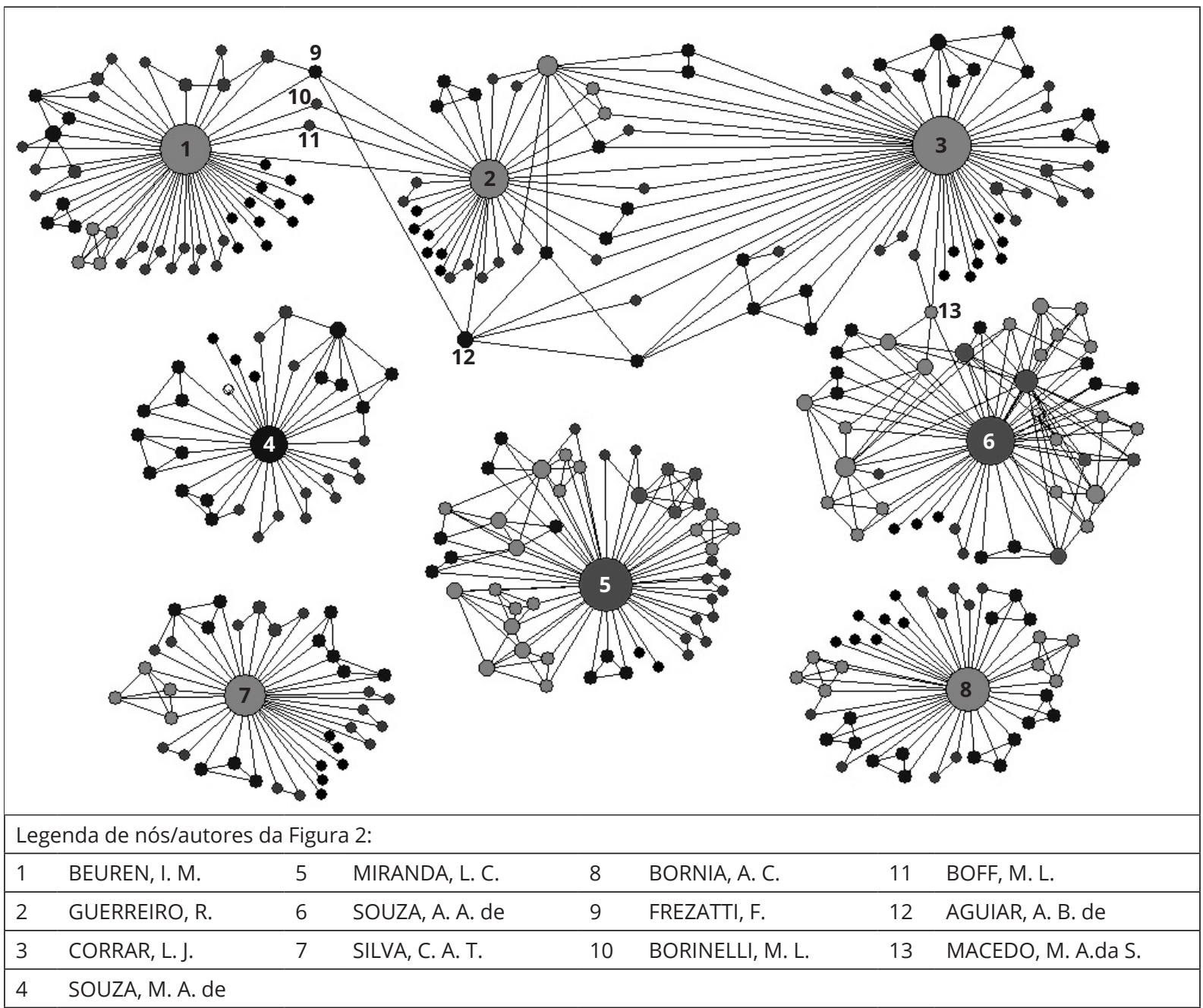

Figura 2. Redes sociais de cooperação entre instituições brasileiras e estrangeiras

Por meio da Figura 2, observa-se que os oitos autores (nós) com maior número de publicações são centrais em suas redes. Esse resultado corrobora o destacado por Shah (2000) de que os atores que permanecem por um amplo tempo em uma rede, como os continuantes apresentados na Figura 2, tendem a tornarem-se centrais. Essa propriedade de centralidade é importante para os pesquisadores, pois reflete a importância desses pesquisadores como principal ponto de referência em suas redes e de articulação entre diferentes informações, pesquisas e pesquisadores (Wasserman \& Faust, 1994). Além disso, Knoke (1990) aponta que a proeminência de um ator em uma rede pode lhe possibilitar influenciar as atitudes e os comportamentos de outros atores desta rede.

Dos oito autores mais prolíficos, quatro - Ilse M. Beuren, Reinaldo Guerreiro, Luiz J. Corrar e Antônio A. de Souza - se conectaram a outro(s) dos oito principais. Reinaldo Guerreiro (2), vinculado à USP, por exemplo, estabeleceu relacionamento tanto com Ilse M. Beuren (1) - que, inicialmente, era vinculada à UFSC e, atualmente, à FURB e que realizou doutorado na USP - quanto com Luiz J. Corrar (3), vinculado à USP. Essas cooperações ocorreram de maneira direta por meio de laços fortes entre os pesquisadores, bem como de forma indireta a partir de pontes ou de laços fracos (Granovetter, 1973). Assim, Ilse M. Beuren e Reinaldo Guerreiro se relacionaram tanto ao publicarem em parceria quanto com autores em comum, como Fábio Frezatti (9), vinculado à USP; Márcio L. Borinelli (10), vinculado, inicialmente, à UEM e, atualmente, à USP; e Marines L. Boff (11), vinculada à FURB. A conexão entre Luiz J. Corrar e Reinaldo Guereiro também é realizada das suas formas, sendo que o número de autores com os quais ambos publicaram é mais amplo. 
Já o relacionamento entre as redes que possuem como atores centrais Luiz J. Corrar (3) e Antônio A. de Souza (6) - vinculado atualmente à UFMG - foi estabelecido apenas por meio de um contato indireto (laço fraco) a partir do autor Marcelo A. da S. Macedo (13), vinculado à UFRRJ (professor) e à USP (pós-doutorado). Nesse caso, observa-se a ocorrência de uma lacuna estrutural, a qual, de acordo com Burt (1992), fornece uma vantagem competitiva para o indivíduo que realiza a conexão, uma vez que esse usufrui acesso às informações das duas redes. Como destacado por Powel, Koput e Smith-Doerr (1996) e Stuart e Podolny (1999), a conexão a uma rede também fornece acesso à inovação proporcionada por diferentes indivíduos. Esse indivíduo também pode conquistar poder de agenciamento de contato entre os autores dos diferentes grupos aos quais se encontra vinculado.

Andson B. de Aguiar (12), vinculado à USP, se destaca nas redes de cooperação apresentadas por estabelecer conexão com três diferentes grupos. Pode-se perceber que ele estabelece parceria em publicação com Luiz J. Corrar (3) e com Reinaldo Guerreiro (2), representados pelos laços fortes, bem como estabelece um laço fraco com a rede que tem como ator central Ilse M. Beuren (1), por meio de Fábio Frezatti (9), vinculado à USP. Dessa forma, Fábio Frezatti também pode, ao estabelecer o diálogo entre esses grupos, desempenhar um papel importante no agenciamento dos contatos e das informações entre os integrantes das três redes, bem como proporcionar inovações e contribuições.

Os demais autores centrais - Marcos A. de Souza (4), vinculado à Unisinos (professor) e à USP (doutorado); Luiz C. Miranda (5), vinculado atualmente à UFPE; César A. T. Silva (7), vinculado à UnB; e Antonio C. Bornia (8), vinculado atualmente à UFSC -, estabelecem laços com diferentes autores de suas redes, mas não se conectam a outros dos oito autores mais prolíficos analisados.

Na Tabela 6, destaca-se a quantidade de laços de cada um dos oito autores mais prolíficos com cada uma das categorias.

Tabela 6

Quantidade de laços por categoria dos autores mais prolíficos

\begin{tabular}{lcccccc}
\hline \multicolumn{1}{c}{ Autores } & Continuantes & Transientes & One-timers & Entrantes & Retirantes & Total \\
\hline BEUREN, I. M. & $34,52 \%(29)$ & $33,33 \%(28)$ & $21,43 \%(18)$ & $2,38 \%(2)$ & $8,33 \%(7)$ & 84 \\
\hline MIRANDA, L. C. & $31,48 \%(34)$ & $10,19 \%(11)$ & $19,44 \%(21)$ & $10,19 \%(11)$ & $28,70 \%(31)$ & 108 \\
\hline CORRAR, L. J. & $56,25 \%(36)$ & $20,31 \%(13)$ & $9,38 \%(6)$ & $3,13 \%(2)$ & $10,94 \%(7)$ & 64 \\
\hline SOUZA, M. A. de & $40,00 \%(24)$ & $20,00 \%(12)$ & $15,00 \%(9)$ & $11,67 \%(7)$ & $13,33 \%(8)$ & 60 \\
\hline SOUZA, A. A. de & $33,67 \%(33)$ & $10,20 \%(10)$ & $18,37 \%(18)$ & $18,37 \%(18)$ & $19,39 \%(19)$ & 98 \\
\hline SILVA, C. A. T. & $25,00 \%(13)$ & $17,31 \%(9)$ & $13,46 \%(7)$ & $7,69 \%(4)$ & $36,54 \%(19)$ & 52 \\
\hline GUERREIRO, R. & $64,71 \%(33)$ & $9,80 \%(5)$ & $7,84 \%(4)$ & $5,88 \%(3)$ & $11,76 \%(6)$ & 51 \\
\hline BORNIA, A. C. & $34,55 \%(19)$ & $12,73 \%(7)$ & $23,64 \%(13)$ & $9,09 \%(5)$ & $20,00 \%(11)$ & 55 \\
\hline
\end{tabular}

A Tabela 6 deixa perceptível que os oito autores mais prolíficos publicam, principalmente, com continuantes, o que reitera sua importância na consolidação do conhecimento científico da área. Ilse M. Beuren, por exemplo, praticamente equilibrou suas publicações entre continuantes e transientes, apresentando o maior percentual de parcerias com a última categoria. Além disso, Ilse M. Beuren exibe o menor percentual de publicações com entrantes.

Luiz C. Miranda publicou, principalmente, com continuantes e retirantes, bem como apresenta o menor percentual de publicações com transientes. Assim, esse autor costuma publicar com os pesquisadores mais tradicionais da área.

Luiz J. Corrar e Marcos A. de Souza publicaram, principalmente, com continuantes. Antônio A. de Souza também se destaca como o autor com maior percentual de parcerias com entrantes. Dessa forma, nota-se que esse autor está atraindo novos pesquisadores para o campo. 
Em contraposição, César A. T. Silva apresenta seu maior percentual de cooperação com autores retirantes. Esse resultado está ligado ao fato de esse autor ser um dos pioneiros da área, apresentando publicações em 15 dos 16 anos analisados (Tabela 5).

Reinaldo Guerreiro exibe o maior percentual de publicações com continuantes e o menor com one-timers, aspecto que se alinha ao identificado nas redes de cooperação (Figura 2) de que esse autor apresenta um grande número de laços fortes com diferentes autores de dois grupos importantes de pesquisadores, tanto o que possui como ator central Ilse M. Beuren quanto o de Luiz J. Corrar.

Por fim, Antonio C. Bornia publica, principalmente, com continuantes, também apresentando, todavia, o maior percentual de parcerias com one-timers.

A Figura 3 apresenta as redes de cooperação internacionais do período (instituições brasileiras que tiveram coautoria com instituições estrangeiras), perfazendo o total de 174 instituições envolvidas.

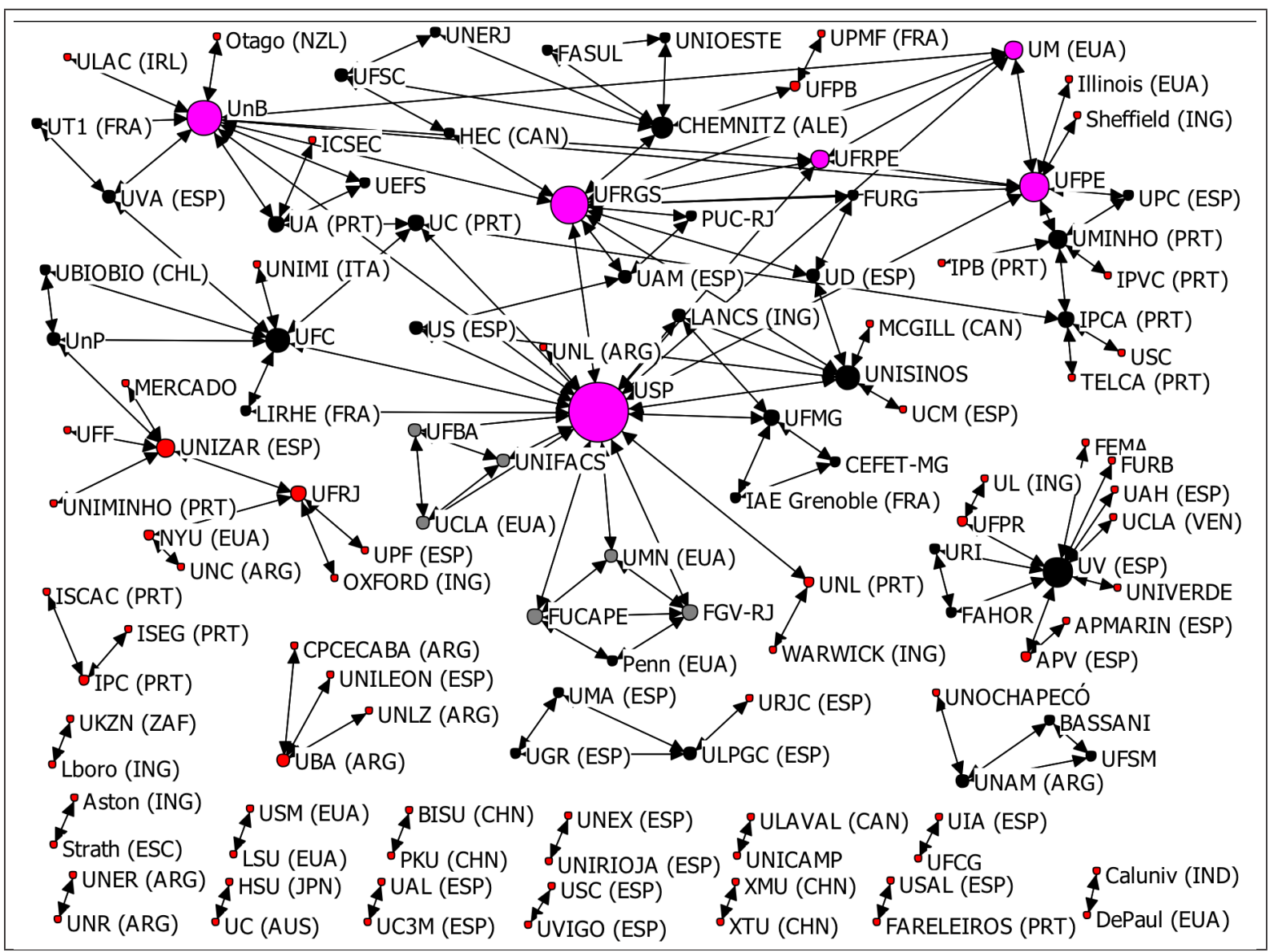

Figura 3. Redes sociais de cooperação entre instituições brasileiras e estrangeiras

A Figura 3 possibilita observar que as 38 instituições nacionais que se associaram a estrangeiras o fizeram com 39 instituições pertencentes a 13 diferentes países, sendo uma alemã, duas argentinas, uma australiana, três canadenses, uma chilena, dez espanholas, seis norte-americanas, quatro francesas, três inglesas, uma irlandesa, uma italiana, uma neozelandesa e cinco portuguesas.

Ressalta-se que, das 128 instituições estrangeiras, 84 se associaram e as outras 44 publicaram de forma isolada. Em relação às cooperações firmadas com instituições brasileiras, percebe-se, no topo da Figura 3, uma ampla rede de cooperação, na qual se destacam como atores centrais a USP, que apresenta 13 laços, em 13 artigos, com 9 instituições do exterior diferentes; a UFRGS, com 8 laços, em 8 artigos, com 5 instituições; a UnB, com 8 laços, em 6 artigos, com 6 instituições; a UFPE, com 6 laços, em 6 artigos, com 
5 instituições; e a Unisinos, com 7 laços, em 7 artigos, com 5 instituições. Tem-se, também, uma rede com uma instituição estrangeira como central, a UV, da Espanha (direita da Figura 3). Essa instituição estabeleceu parcerias com seis instituições do Brasil, bem como com outras três estrangeiras.

A análise do panorama geral de internacionalização evidencia que a amplitude de associações de cada instituição brasileira com universidades estrangeiras ainda é limitada. Entretanto, devido à riqueza de possíveis trocas de conhecimento com universidades e pesquisadores estrangeiros, há um amplo espaço para crescimento dessas associações.

No que se refere às categorias de produção e de continuidade dos autores que realizaram parcerias internacionais, ressalta-se que se classificaram $42(33,07 \%)$ como continuantes, $23(18,11 \%)$ como transientes, 27 (21,26\%) como one-timers, 19 (14,96\%) como entrantes e 16 (12,6\%) como retirantes, totalizando 127 autores que publicaram com autores do exterior. Esse resultado reitera a importância dos autores continuantes para o amadurecimento e desenvolvimento de um campo científico também no que concerne à troca de informações e ao desenvolvimento de cooperações internacionais.

\section{Considerações finais}

Esta pesquisa buscou verificar, sob uma perspectiva longitudinal e de acordo com categorias de produção e de continuidade, o papel desempenhado pelos autores no desenvolvimento da produção científica brasileira em contabilidade. Os resultados obtidos demonstraram a importância dos autores classificados como continuantes para o desenvolvimento da pesquisa em contabilidade e, consequentemente, para a consolidação e o amadurecimento dessa área do conhecimento. Isso porque esses autores apresentaram a maior produtividade quantitativa entre as categorias, demonstrando seu potencial na difusão do conhecimento da área contábil e no desenvolvimento de pesquisas que visam ampliar o conhecimento acumulado nesse campo.

Além disso, observou-se que os continuantes atuam intermediando o relacionamento com diferentes categorias, visto que o maior índice de coautorias das demais categorias é com continuantes, ou seja, estes atuam agenciando as informações e os conhecimentos de diferentes grupos de pesquisadores. As parcerias com transientes, por exemplo, refletem o fluxo de informações mais estáveis da rede. Já as publicações com retirantes indicam que estes possuem acesso aos conhecimentos mais tradicionais. Em contraposição, ao pesquisarem ao lado de entrantes e on-timers, os continuantes podem ter acesso a inovações e a novas perspectivas para o tratamento do conhecimento do campo. Corroborando esse resultado, tem-se, ainda, que os oitos autores continuantes com maior número de publicações são centrais em suas redes. Dessa forma, esses autores podem atuar articulando as pesquisas e os conhecimentos vinculados a diferentes pesquisadores da área.

Outro aspecto que demonstra o importante papel desempenhado pelos autores continuantes situa-se no fato de essa categoria ser a principal responsável pelas parcerias internacionais realizadas. Nesse sentido, reitera-se, também, o grande espaço existente para a realização de parcerias com autores do exterior, visto que tais parcerias ainda se apresentam restritas e que diversas instituições estrangeiras realizaram publicações no Brasil (com ou sem parcerias nacionais), o que demonstra o interesse dessas entidades em publicar no país.

Apesar da importância dos autores continuantes para o desenvolvimento do campo de pesquisa em contabilidade, notou-se que eles representam o menor percentual entre as categorias. Em contraposição, o maior número de autores identificados classificou-se como one-timer, ou seja, realizou uma única publicação em todo o período. Essa última categoria, aliada aos entrantes, indica a atratividade exercida pela área do conhecimento a pesquisadores, podendo consistir nas principais fontes de inovações e de novas abordagens. Contudo, os entrantes apresentam uma tendência a se estabilizarem no campo da contabilidade, passando a oferecer uma contribuição mais ampla para seu desenvolvimento. 
Por fim, em relação aos retirantes, observou-se certa rotatividade, o que é natural em todos os campos da pesquisa. Todavia, é salutar que esse percentual não seja superior ao número de pesquisadores atraídos para a área. Assim, apesar de a parcela de retirantes, nesta pesquisa, ser maior que a de entrantes, é provável que alguns dos pesquisadores considerados como one-timers nos períodos mais recentes se configurem como entrantes em breve.

Como limitação desta pesquisa, destaca-se a amostra focada em eventos, a qual poderia sem ampliada para considerar também periódicos. Para futuras pesquisas sugere-se verificar se os resultados deste estudo se confirmam, também, nas subáreas da contabilidade.

\section{Referências}

Araujo, E. A. T., Oliveira, V. do C. \& Silva, W. A. C. (2009). Estudo bibliométrico da produção científica sobre contabilidade gerencial. Anais do Seminários em Administração, São Paulo, SP. FEA/USP, 12.

Braun, T., Glänzel, W. \& Schubert, A. (2001). Publication and cooperation patterns of the authors of neuroscience journals. Scientometrics, 51(3), 499-510.

Burt, R. S. (1992). Structural holes: the social structure of competition. Cambridge, MA: Havard University Press.

Cardoso, R. L., Mendonça Neto, O. R.; Riccio, E. L. \& Sakata, M. C. G. (2005). Pesquisa científica em contabilidade entre 1990 e 2003. Revista de Administração de Empresas, 45(2), 34-45.

Espejo, M. M. dos S. B., Cruz, A. P. C. da, Walter, S. A. \& Gassner, F. P. (2009). Campo de pesquisa em contabilidade: uma análise de redes sob a perspectiva institucional. Anais do Encontro da Associação Nacional dos Programas de Pós-graduação em Ciências Contábeis e International Accouting Congress,. São Paulo, SP, ANPCONT, 03

Galaskiewicz, J.\& Wasserman, S. (1994). Advances in social network analysis: research in the social and behavioral sciences. London: Sage.

Gordon, A. (2007). Transient and continuant authors in a research field: the case of terrorism. Scientometrics, 72(2), 213-224.

Granovetter, M. (1973). The strength of weak ties. American Journal of Sociology, 78(6), 1360-1380.

Guarido Filho, E. R., Machado-da-Silva, C. L. \& Gonçalves, S. A. (2009). Institucionalização da teoria institucional nos contextos dos estudos organizacionais no Brasil. Anais do Encontro da Associação Nacional dos Programas de Pós-graduação em Administração, São Paulo, SP. ANPAD, 33.

Knoke, D. (1990). Political networks: the structural perspective. New York: Cambridge University Press.

Leite Filho, G. A. (2006). Padrões de produtividade de autores em periódicos de congressos na área de contabilidade no Brasil: um estudo bibliométrico. Anais do Congresso USP de Controladoria e Contabilidade, São Paulo, SP, FEA/USP, 6.

Liu, X.; Bollen, J., Nelson, M. L. \& Van de Sompel, H. (2005). Coauthorship networks in the digital library research community. Information Processing \& Management, 41(6), 1462-1480.

Macias-Chapula, C. A. (1998). O papel da informetria e da cienciometria e sua perspectiva nacional e international. Ciência da Informação, 27(2), 134-140.

Marsden, P. V. (1993). The reliability of network density and composition measures. Social Networks, 15(4), 399-421.

Mendonça Neto, O. R. , Cardoso, R. L., Riccio, E. L. \& Sakata, M. C. G. (2004). Estudo sobre as publicações científicas em contabilidade: uma análise de 1990 até 2003. Anais do Encontro da Associação Nacional dos Programas de Pós-graduação em Administração, Curitiba, PR, ANPAD, 28,. 
Oliveira, M. C. (2002). Análise dos periódicos brasileiros de contabilidade. Revista Contabilidade \& Finanças, 13(29), 68-86.

Powell, W. W., Koput, K. W. \& Smith-Doerr-DOERR, L. (1996). Interorganizational collaboration and the locus of innovation: networks of learning in biotechnology. Administrative Science Quarterly, 41(1), 116-145.

Riccio, E. L., Sakata, M. G. \& Carastan, J. T. (1999). Accounting research in brazilian universities: 19621999. Caderno de Estudos da FIPECAFI, 10(22), 35-44.

Shah, P. P. (2000). Network destruction: the structural implications of downsizing. Academy of Management Journal, 43(1), 101-13.

Silva, B. M. dos S. da \& Ensslin, S. R. (2008). O perfil dos artigos publicados nos congressos de iniciação científica em contabilidade da UFSC e da USP: um estudo bibliométrico. Anais do Congresso UFSC de Iniciação Científica em Contabilidade, Florianópolis, SC. 2.

Silva, P. Y. C. da \& Pires, J. S. D. B. (2009). Investigação e análise da produção científica em contabilidade e sociedade no Brasil nos últimos 20 anos. Anais do Encontro Internacional de Produção Científica CESUMAR, Maringá, PR, 6.

Smith-Doerr, L. \& Powell, W. W. (2003). Networks and economic life. In N. Smelser \& R. Swedberg. (Eds.). The handbook of economic sociology. Boston: Sage.

Stuart, T. E. \& Podolny, J. M. (1999). Positional consequences of strategic alliances in the semiconductor industry. In S. B. Andrews, S. B. \& D. Knoke (Eds.). Research in the Sociology of Organizations, Vol. 16. Stamford, Co.: JAI Press. pp. 161-182.

Souza, F. C. de; Rover, S.; Gallon, A. V. \& Ensslin, S. R. (2008). Análise das IES da área de ciências contábeis e de seus pesquisadores por meio da produção científica. Revista Contabilidade Vista \& Revista, 19(3), 15-38.

Walter, S. A. \& Silva, E. D. da. (2008). Visão baseada em recursos: um estudo bibliométrico e de redes sociais da produção científica da área de estratégia do EnANPAD 1997-2007. Anais do Encontro da Associação Nacional dos Programas de Pós-graduação em Administração, Rio de Janeiro, RJ, ANPAD, 32.

Walter, S. A., Cruz, A. P. C., Espejo, M. M. S. B. \& Gassner, F. P. (2009). Uma análise da evolução do campo de ensino e pesquisa em contabilidade sob a perspectiva de redes. Anais do Congresso USP Controladoria e Contabilidade, São Paulo, SP. FEA/USP. 10.

Walter, S. A., Lanza, B. B. B., Sato, K. H., Silva, E. D. da \& Bach, T. M. (2010) . Análise da produção científica de 1997 a 2009 na área de estratégia: produção e continuidade de atores e cooperação entre instituições brasileiras e estrangeiras. Anais do Encontro da Associação Nacional dos Programas de Pós-graduação em Administração, São Paulo, SP, ANPAD, 34.

Wasserman, S. \& Faust, K. (1994). Social network analysis: methods and applications. Cambridge University Press.

White, H. C., Boorman, S. A. \& Breiger, R. L. (1974). Social structure from multiple networks. American Journal of Sociology, 81(4), 730-780. 